\title{
Investigation of wireless magnetometer in sensing magnetic field changes at different car direction and speed
}

\author{
Chin Fhong Soon ${ }^{1}$, Siti Hajar Aminah $\mathrm{Ali}^{2}$, See Khee $\mathrm{Yee}^{3}$, Munzilah Md Rohani ${ }^{4}$, Kian Sek Tee, \\ Marlia Morsin ${ }^{6}$, Nafarizal Nayan ${ }^{7}$, Chiok Chuan Lim ${ }^{8}$ \\ ${ }^{1,6,7}$ Microelectronics and Nanotechnology-Shamsuddin Research Centre, Institut for Integrated Engineering, Universiti \\ Tun Hussein Onn Malaysia, Johor, Malaysia \\ 1,2,3,5Faculty of Electrical and Electronics Engineering, Universiti Tun Hussein Onn Malaysia, Johor, Malaysia \\ ${ }^{3}$ Research Center for Applied Electromagnetic, Institute for Integrated Engineering, Universiti Tun Hussein Onn \\ Malaysia, Johor, Malaysia \\ ${ }^{4}$ Faculty of Civil Engineering and Built Environment, Universiti Tun Hussein Onn Malaysia, Johor, Malaysia. \\ ${ }^{8}$ Sena Traffic System Sdn. Bhd., Selangor, Malaysia
}

\begin{tabular}{l} 
Article Info \\
\hline Article history: \\
Received Jun 11, 2020 \\
Revised Jun 23, 2020 \\
Accepted Aug 18, 2020 \\
\hline
\end{tabular}

Keywords:

Coordinate axis

Magnetic field

Magnetometer

Sensor

Vehicle detection

Wireless

\begin{abstract}
The embedment of induction loop underground for traffic volume monitoring caused damaging effects to the road and reduced road surface aesthetics. A wireless magnetometer implanted underground in a small uniform area was developed to detect three-axis magnetic flux changes due to the perturbation of vehicle passing over the sensor. In this project, a wireless magnetometer sensor system operating at a radio frequency of $2.4 \mathrm{GHz}$ for detecting and transmitting Z-field data has been developed to investigate the patterns of magnetic field associated with the car directions and speed. This is the first report in revealing the responses of the sensor to different car speed and sensing directions. Field tests were conducted by car passes over in a direction in-line or countering the $\mathrm{X}$ and $\mathrm{Y}$ axes of the magnetometer. The results showed that the strong magnetic field density as low as -100 to -230 $\mu \mathrm{T}$ could be generated when a car passed over the sensor in a direction countering $\mathrm{X}$ and $\mathrm{Y}$ axes. The speed detection limit of the sensor is $<60$ $\mathrm{kmph}$. The $\mathrm{X}, \mathrm{Y}$ and $\mathrm{Z}$ flux patterns obtained is important in designing an algorithm for accurate detection and counting of vehicles.
\end{abstract}

This is an open access article under the CC BY-SA license.

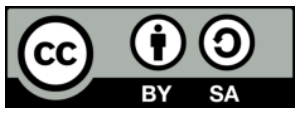

\section{Corresponding Author:}

Chin Fhong Soon

Microelectronics and Nanotechnology-Shamsuddin Research Centre

Institut for Integrated Engineering, University Tun Hussein Onn Malaysia

Parit Raja, 86400 Batu Pahat, Johor, Malaysia

Email: soon@uthm.edu.my

\section{INTRODUCTION}

Underground electrical wire used as the induction-loop or piezoelectric traffic sensor is the present technique [1] commonly used to detect the presence of a vehicle, in which, the changes of magnetic field in the induction-loop sensors embedded underground are send via the electrical wire to an electrical meter and computer system. This technology can be used for counting vehicles or traffic-actuated signal control. This technology is applied for collecting road statistics and solving traffic congestion problem [2, 3]. The monitoring system sends the data from sensors to the controller through the wire planted underground. Then, the intelligence of the traffic controller would mitigate the congestion at the junctions through the traffic lights control $[4,5]$. Other applications of induction loop are such as detecting vehicles driving in the wronglane and wrong-way which has cause fatal road accidents in United State of America [6, 3]. The 
disadvantages of this system are the requirements of digging long un-uniform ridges in the surface of the road to implant the induction-loop wire and the excavation of the asphalt concrete caused damaging effects to the surface of the road. In addition, traffic measurement system based on vision processing system [7] faces the challenges of highly variable brightness of images during day and night time which required a highly adaptable algorithm for subtracting the background image at different time of a day. Hence, the existing vehicle counting system could be improved by implanting a few wireless magnetometer sensors that could send the data of the detected vehicles wirelessly to a gateway placed in the traffic controller cabinet. Such system may ease the monitoring of traffic at the centrally managed Internet of Things (IoT) system. Subsurface sensor node is always an attractive solution for environment [8] and traffic system [9].

A magnetometer is an electronic device which could measure local earth magnetic field direction in space. There are several types of magnetometer such as fluxgate, magneto-inductive, and magneto-inductive [10-15]. The magnetometer applies a passive sensing technology to sense large ferrous objects. The introduction of ferrous objects induces change in the ambient magnetic field. Hence, the wireless magnetometer maybe an alternative replacement for inductive loop systems. The sensor node consists of a robust and solid housing contains the power source, sensor, and antenna for a completely wireless solution. It is possible to distort this uniform field by placing a 'highly permeable' metal in this field. Under highly permeable field induction, the ferrous metals allow maximum amount of flux lines corresponding to an external magnetic field to pass through them. The ferrous material can be easily magnetized by exposing to an external applied magnetic field. Before a vehicle approaches the magnetometer sensor, the sensor would experience uniform field. When a ferrous body such as a car moved over the magnetometer and cuts through the earth's field, the flux lines are absorbed into the metal, creating non-uniformity in the flux line distribution. As the vehicle ran over the sensor node, it distorts the earth's magnetic field and creates an increase and decrease flux line concentration. The resulting time responses of the magnetic flux density or "signature response" indicate that a vehicle had passed over the magnetometer sensor [16].

The responses of the magnetic flux to different car speed and direction of measurement by the magnetometer sensor rarely been discussed. A few issues such as false detection of adjacent vehicle [17], car crossing the wrong lane and double counting were reported in [10] but the detection range and anisotropic behavior of the magnetic sensor were not investigated. The study in our project clarified the detection limit of magnetometer by revealing the fundamental characteristic of the magnetometer in detecting different speed and passage direction of a car. This is the first report in revealing the details of dynamic magnetic field data generated by a 3 -axis magnetometer in detecting moving vehicle at various speed $(5-60 \mathrm{kmph})$ and sensing vehicle associated with to the coordinate system of the sensor. The objective of this project is to identify the magnetic flux responses of a wireless magnetometer sensor node to car passes in different direction and speed. This project also presents a customized graphical user interface software to display the information received from the wireless sensor node. In practical application, the magnetometer can be placed in any direction on the road relative to the magnetic field of the earth. Hence, it is of paramount importance to understand the effects of positioning the magnetometer in response to vehicle passes over in different direction and speed.

The project was initiated with fundamental test of the magnetometer in response to a ferrous object passing over, inclusion of the wireless modules, the design of the program in the microcontroller, halting the car on the magnetometer and field test for sensor direction and car speed detection. The developed wireless vehicle detection system consists of a wireless magnetometer transmitter, a gateway and graphical user interface (GUI) display. The magnetometer sensor will be planted underground, and the flux intensity modulated due to the interaction with the car will be measured wirelessly using $2.4 \mathrm{GHz}$ transceivers and reported. The contributions of this project are in revealing the detection limit of the magnetometer to a range of car speed and positioning of magnetometer for strongest signal of detection.

\section{RESEARCH METHOD}

\subsection{Fundamental test of the magnetometer in response to ferrous object passing over}

A three-axis electronic magnetometer sensor (HMC5883L module) was used to interface to a Arduino microcontroller and the data output of the magnetometer was decoded by an Analog-to-Digital converter of the microcontroller as digital output to the Arduino Serial Plotter software (Version 1.8.5). The microcontroller reads the data send from the magnetometer via the $\mathrm{I} 2 \mathrm{C}$ protocol using the Wire library. In the program, two registers per axis from address 6 consists of two bytes of data for $\mathrm{X}, \mathrm{Y}$ and Z, respectively were requested from the magnetometer as shown in Figure 1. The sketch used to read the digital value is as shown in Figure 1. A Wire library was used to request data from register number three in which, $\mathrm{X}$ is the most significant byte for the register and followed by $\mathrm{Y}$ and $\mathrm{Z}$ data. Then, the data will be sent via serial protocols to the computer using the Serial.print() command as shown in Figure 1. 
In the investigation on the perturbation of the earth magnetic field measured by the magnetometer, an iron rod was used to move over the HMC5883L magnetometer sensor. The direction of the rod movement was either in-line or counter to the $\mathrm{X}$ and $\mathrm{Y}$ coordinate system of the sensor as illustrated in Figure 2 . The real-time $\mathrm{X}, \mathrm{Y}$ and $\mathrm{Z}$ data measured by the magnetometer sensor was transmitted via the serial port to the plotter software to be profiled. Then, the data was recorded and analyzed using a computer.

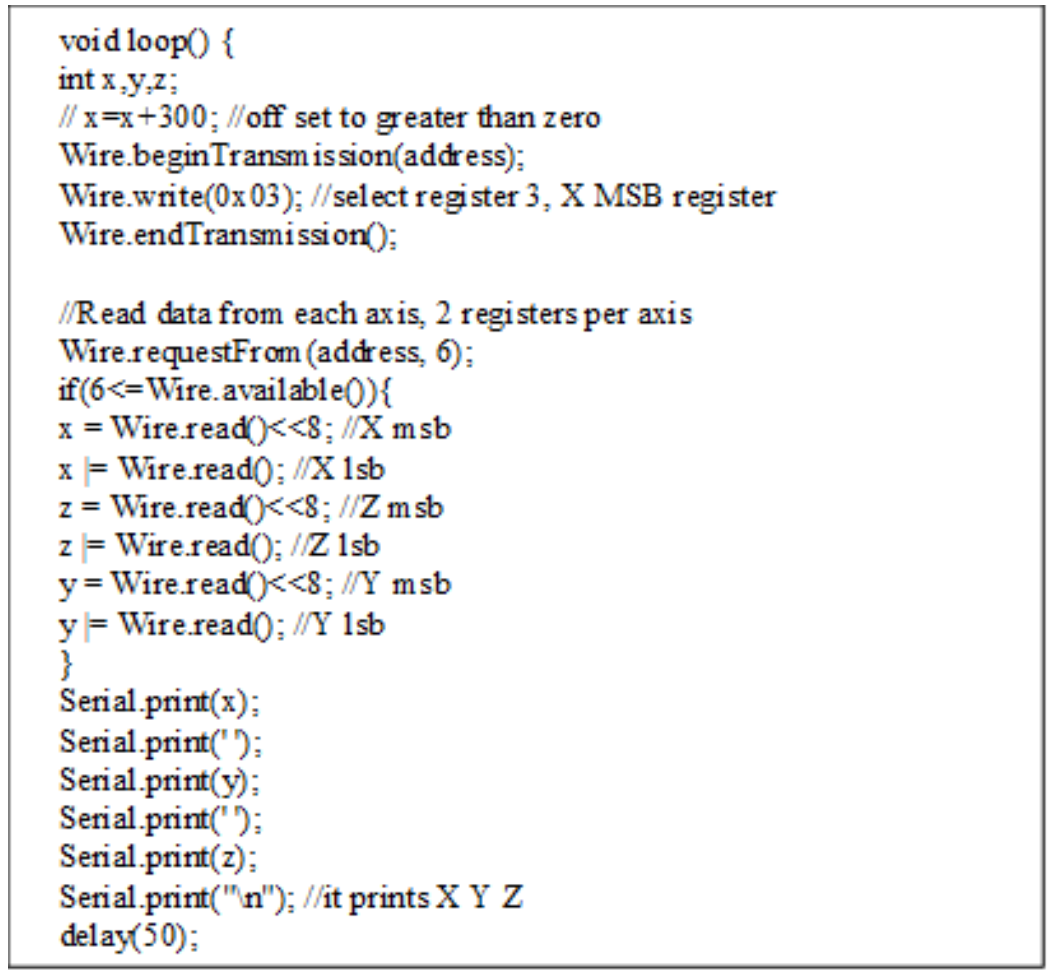

Figure 1. Source code for reading X, Y and Z data from the HMMC 5883L magnetometer sensor

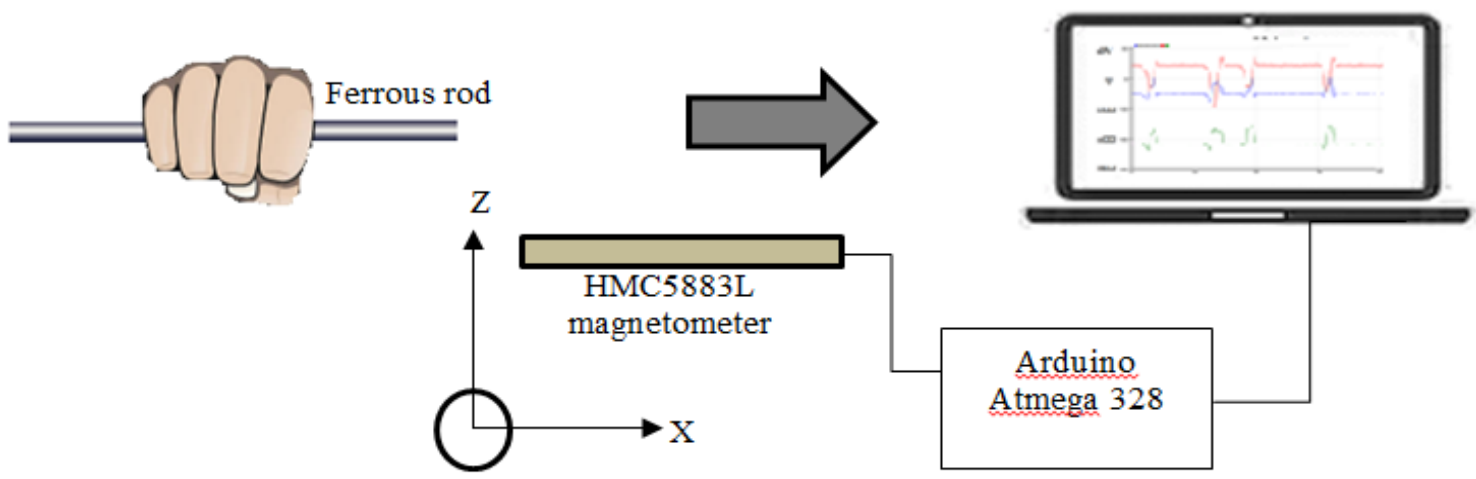

Figure 2. Moving a ferrous rod and acquisition of magnetic field changes via serial plotter software

\subsection{Design of a wireless magnetometer sensor node}

The wireless magnetometer sensors network system consists of three layers: the magnetometer sensor node, wireless transceiver and gateway with data logger, and the computer console Figure 3 . A threeaxis electronic magnetometer sensor (HMC5883L module) was used to interface to a microcontroller via the I2C communication protocol at $+3.3 \mathrm{~V}$ Figure 3 . When the magnetic field of the sensor is interfered by a ferrous object, the magnetic strength in X-Y-Z vectors will provide different readings ( $\mathrm{x}, \mathrm{y}$ and $\mathrm{z}$ data) from the default up to $\pm 800 \mu \mathrm{T}$. From the microcontroller, the digital values of the magnetic field intensity will be 
transferred to a Semitron's SEM-YL-4432G transceiver module of $2.4 \mathrm{GHz}$ for wireless data transmission at a transmission rate of $256 \mathrm{kbps}$ and maximum output power of $20 \mathrm{dBm}$. The output power is enough to ensure extended range and improved link performance. In this module, built-in antenna support for frequency hopping and extended transmission range. Whenever the flux line of the magnetometer is disturbed and sensed, the microcontroller unit transfers the detected value to the transceiver, followed by transmission of the flux data via the transceiver. The microcontroller was programmed to send the Z-field or magnetic field density determined in the Z-axis every $100 \mathrm{~ms}$. This is the minimum sampling rate of the magnetometer to sense and process the flux detected. In this work, the Z-field is of interest and measured for comparison.

The Z-field, NetworkID and DeviceID received by the receiver gateway will be decoded by the microcontroller and send via the UART communication to the graphical user interface (GUI) designed using Python programming language. Then, the raw data recorded with time stamp using the GUI was saved in .csv format and plotted using Microsoft Excel software
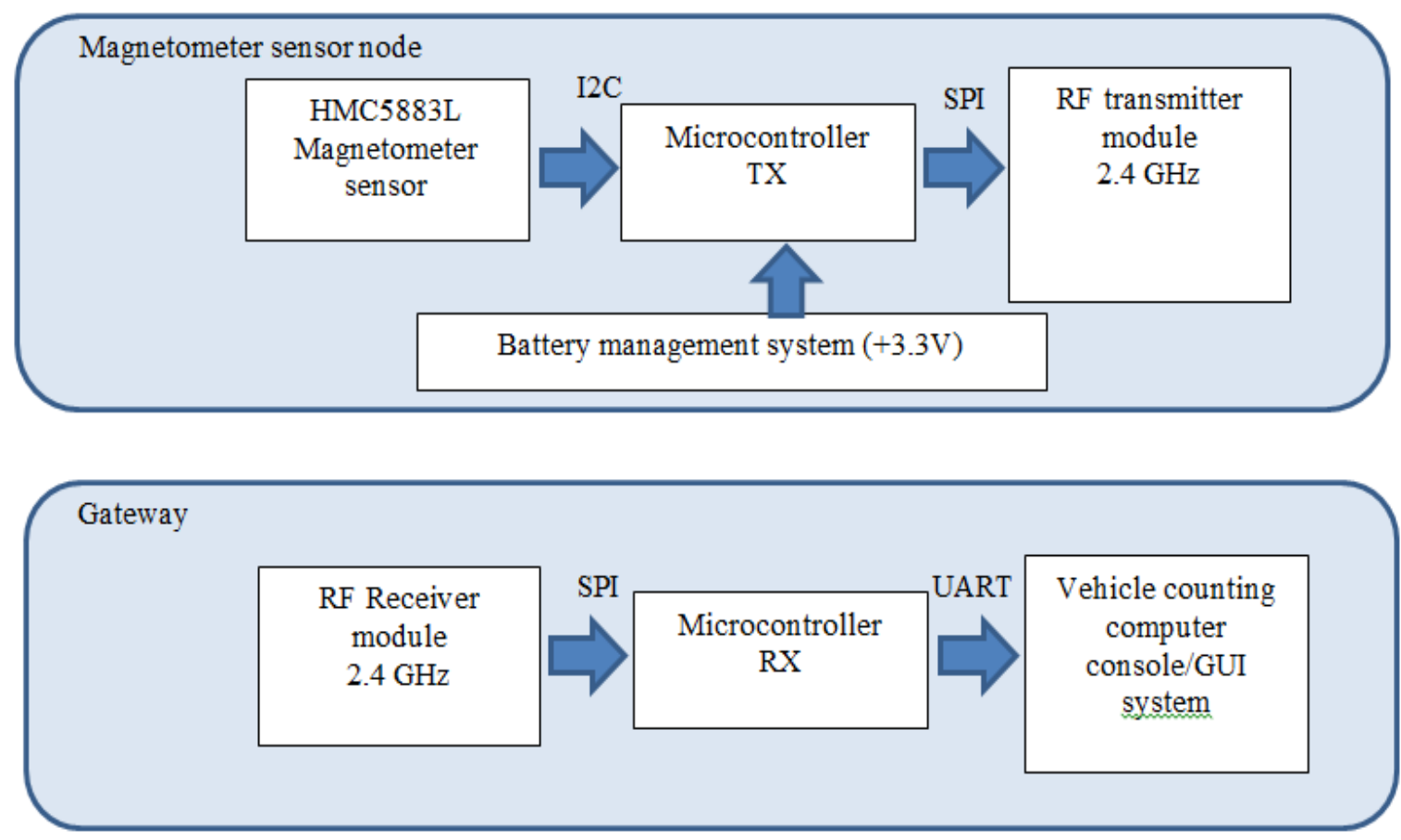

Figure 3. Block diagrams of a wireless magnetometer sensor node and gateway

\subsection{Flow diagram of the algorithm for transmission, receiving network and $Z$-field data}

The Atmega 2560 microcontroller of the magnetometer sensor was programmed in $\mathrm{C}$ language in the Integrated Design Environment (IDE) of Arduino version 1.8.2 software. In the TX microcontroller which communicates with the transmitter and sensor, the program begins with initialization of library for the network Figure 4, magnetometer sensor and radio channel. The program puts the data that was read from the HMMC5883L magnetometer sensor in a structure consisting a payload of address (or deviceID), network, Z and count of vehicle ready to be transferred to the RF module in I2C format. Subsequently, the payload with be attached with a header and send via the Serial Peripheral Interface (SPI) protocol to the RF module for transmission as shown in Figure 4a.

At the receiver, similar initializations for radio channel, network and declaration of variables were performed as shown in Figure 4b. In the initialization, serial, SPI, radio channel and network will be setup accordingly. Then, the algorithm reads the payload while the network availability is updated. After reading the data from the receiver, the microcontroller starts sending data via UART protocol to the GUI written in Python. In the GUI the data will be read and sorted to break the packet of the data into the network ID, device ID, Z-field and vehicle counted to be displayed and updated. The data refresh time was approximately every second. 


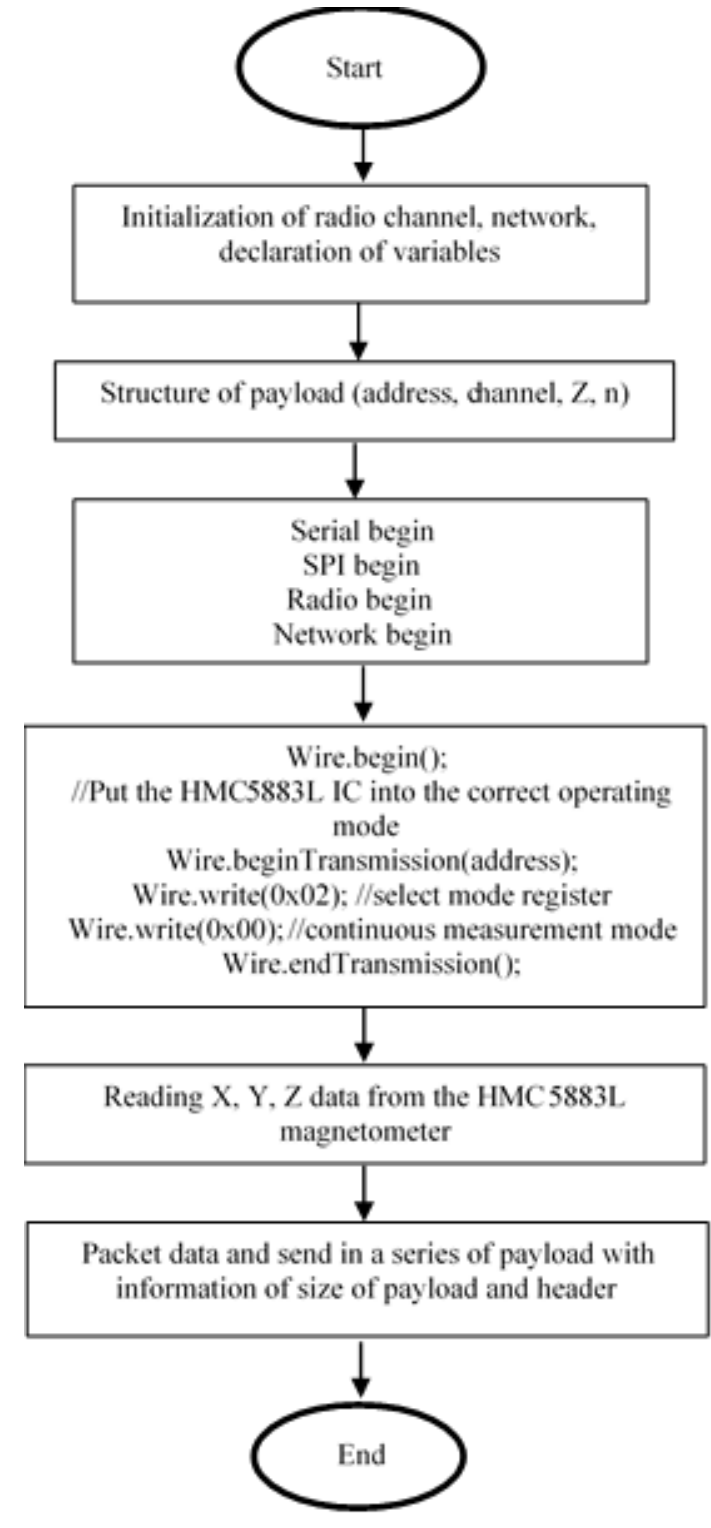

(a)

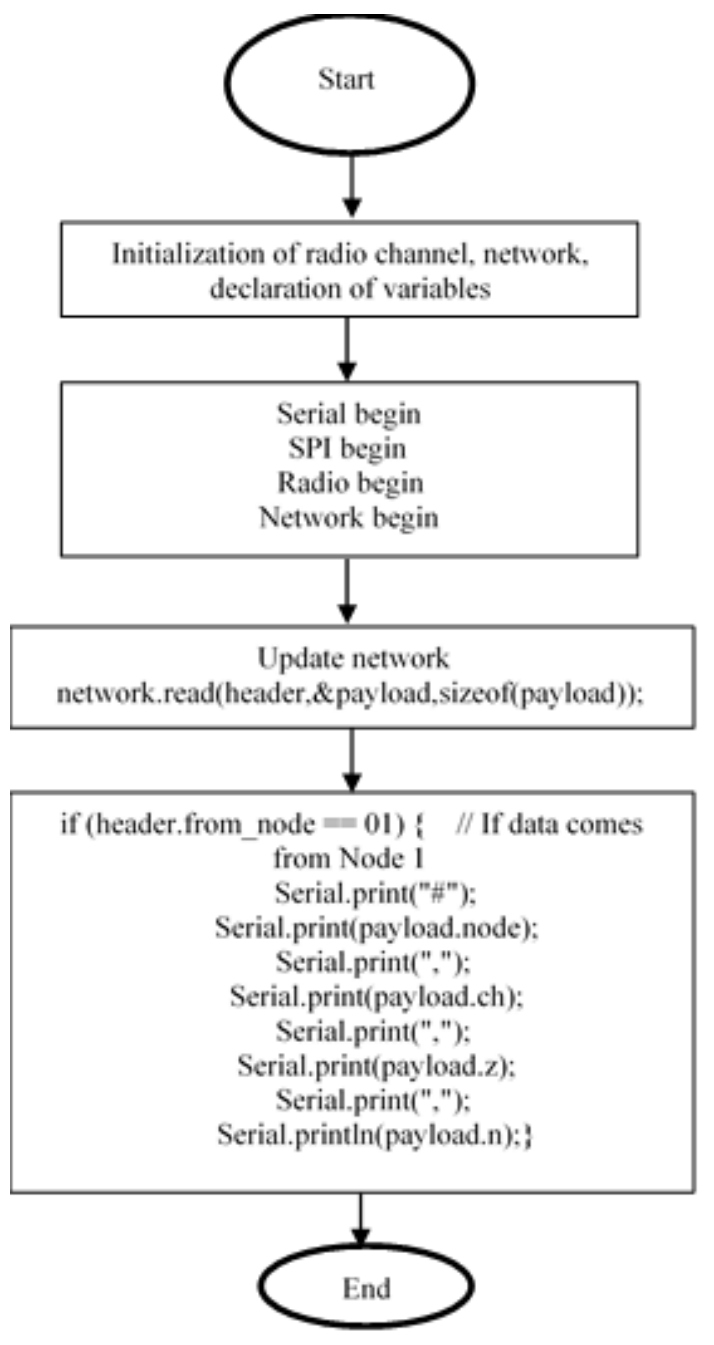

(b)

Figure 4. Flow diagram of the algorithm for (a) payload transmission, (b) reception at the RF modules

\subsection{Experimental setup for $Z$-field data measurement of vehicle passes in-line and countering to $X$ and $\mathrm{Y}$ directions}

In this experiment, a Toyota car was driven to pass over a wireless magnetometer sensor node at a speed of $5 \mathrm{kmph}$ in a direction which is in-line or countering the $\mathrm{X}$ or $\mathrm{Y}$ coordinate system of the magnetometer as shown in Figure 5. The changes of the Z-field will be measured and transmitted wirelessly to the gateway located approximately 10 meters away. The distance between the implanted wireless sensor node to the bottom of the car was approximately $30 \mathrm{~cm}$. The data sampling rate of the magnetometer sensor node was approximately 8 samples per second. The test was conducted in a guarded open field with all the safety rules complied. The format of the data transmitted is in the form of: \#NetworkID, DeviceID, Z-field and vehicle counted. These data will be transmitted and decoded by the receiver and microcontroller at the gateway. Via the UART protocol, the data will be transferred and sorted by the software and the information would be updated on the graphical user interface continuously as shown in Figure 6. The GUI was developed using Python programing language in IDLE software. In the software, the data can be recorded and added with the time stamp in a Microsoft Excel file in *.xls format for plotting into a graph which is associated with the modulation of the magnetic field density by the vehicle. 


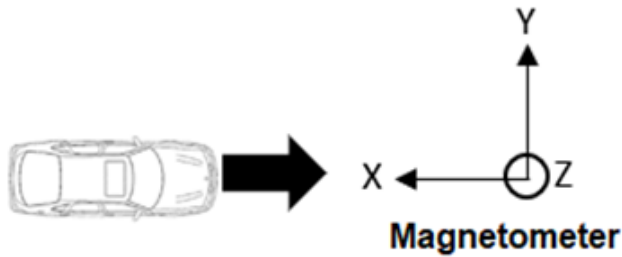

(a)

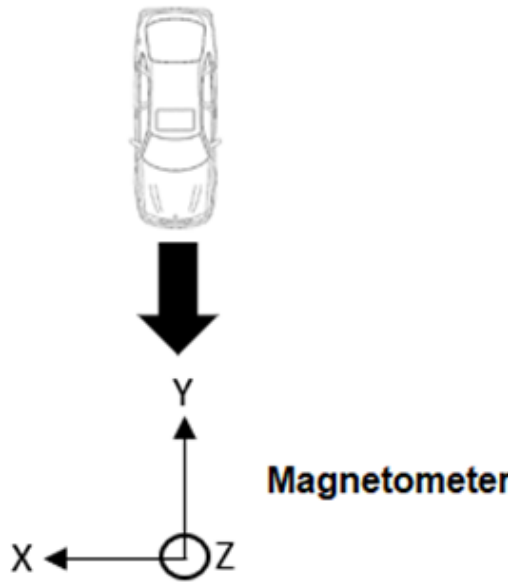

(c)

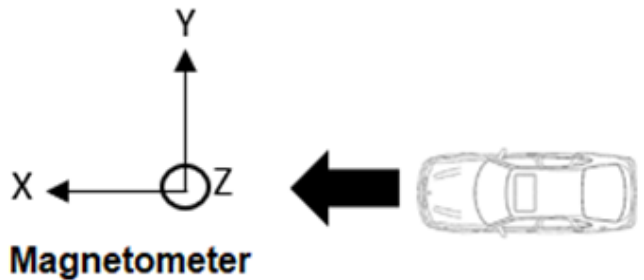

(b)

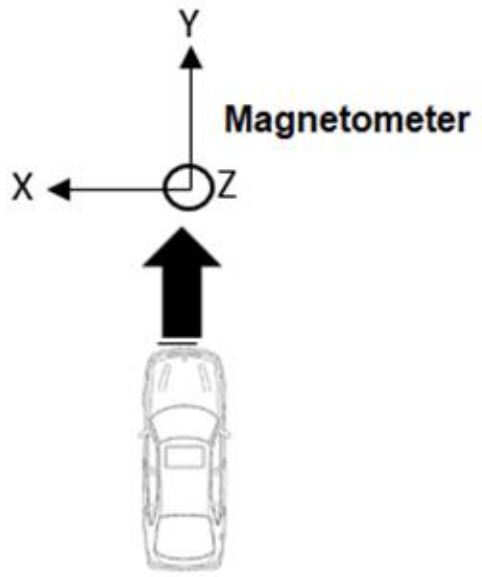

(d)

Figure 5. Graphical depiction of a car passes through an electronic magnetometer in (a) counter X-axis, (b) in-line with $\mathrm{X}$-axis, (c) counter $\mathrm{Y}$-axis and (d) in-line with $\mathrm{Y}$-axis of the measurement coordinate system of magnetometer.

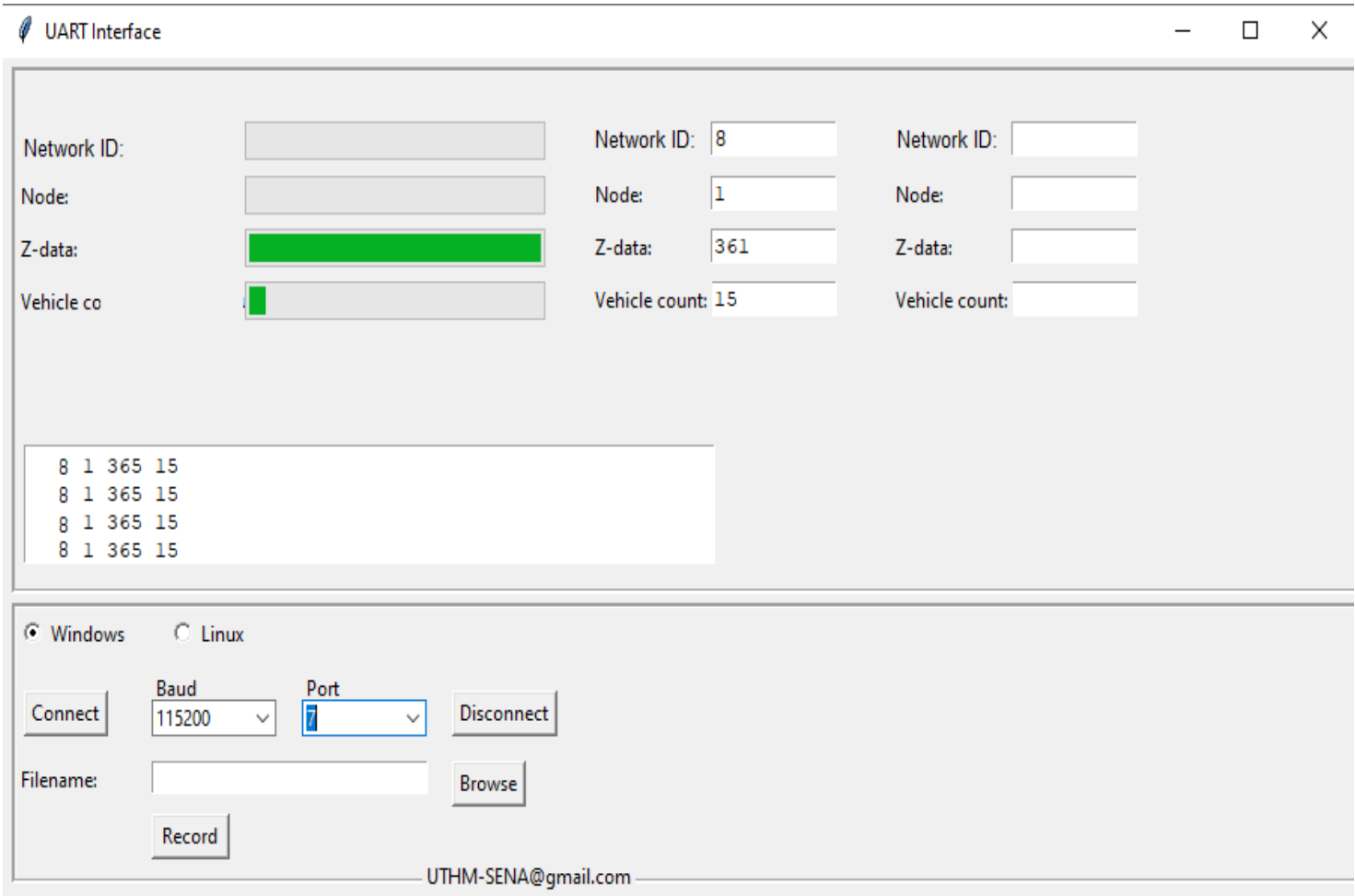

Figure 6. Graphical user interface of the wireless received data from the magnetometer sensor node 


\subsection{Investigating the effects of vehicle halting on the magnetometer sensor node to the magnetic field}

When the magnetometer sensor node is used in practical situation, there is a possibility that a vehicle might stop over the sensor node during heavy traffic congestion. This information is important when designing the algorithm for the microcontroller and sensor in detecting and counting the vehicles passing over. In addition, it is of interest to find out if the ferrous body of the vehicle might block the signal transmission to the receiver unit. Hence, the vehicle was driven over magnetometer sensor node with the engine remaining powered and halted for 5 seconds before moving away from the sensor node. This is to acquire the signal pattern of the magnetic field and putting consideration in designing a device for different practical situation and application.

\subsection{Investigating the detection limit of the magnetometer sensor to different car speed}

The time responses of the magnetometer were recorded by testing the detection limit of the sensor to the car speed set at 5,10,20, 40,40,50 and $60 \mathrm{kmph}$. The magnetometer sensor was planted underground, and the car was initiated in countering Y-axis direction from a starting point which is 100 meters away from the sensor node. Then, the car was driven to pass over the sensor node at a constant speed set. The gateway was placed 10 meters away from the sensor node for continuous measurements. This experiment was performed in an open field abiding to road safety measures.

\section{RESULTS AND ANALYSIS}

For the fundamental test of the magnetometer, it was found that the magnetometer is highly sensitive and generate inverting, non-inverting or biphasic magnetic field patterns in response to ferrous object passing over the sensor which is depending on the direction of the object and the coordinate system of the sensor Figure 7. When a ferrous material with a dipole magnetic field enters a magnetometer's detection zone, this creates magnetic anomaly as detected by the sensor. The dipole magnetic field added the vector field into the quiescent earth's magnetic field produces the magnetic anomaly [1] that were observed as the different magnetic field patterns as shown in Figure 7. These patterns are the magnetic signature of the vehicle [1]. For every single passing over of the iron rod, a single pulse will be generated. When the moving direction of the iron rod is in-line with the $\mathrm{X}$-axis of the magnetometer coordinate system, inverted pulses were generated for Y-field. In comparison, biphasic pulses will be generated when the iron-rod passing over was in the countering the X-axis of the sensor (Figure 7a). Similar non-inverted pulses were generated for the $\mathrm{X}$ and $\mathrm{Z}$. The $\mathrm{X}$ and $\mathrm{Z}$ in $\mu \mathrm{T}$ were always less than zero. However, when the iron rod was moved in a direction in-line the $\mathrm{Y}$-axis of the sensor, biphasic pulses were generated for Y-field (Figure 7b). However, similar biphasic pulses were detected when the iron-rod was moved over in a direction countering the Y-axis of the sensor. The $\mathrm{X}$ and $\mathrm{Z}$ data remained as non-inverted pulses (Figure 7c and d). For all the measurements regardless of direction of rod movement, the default $Y$-data remained in positive field values. Stronger pulses were generated when the iron-rod was tested in-line and countering $\mathrm{Y}$-axis of the sensor.

The iron bar or the vehicle disturbed the magnetic flux lines exist around the magnetometer sensor because ferrous materials are more permeable to magnetic flux than air [1]. As the vehicle moved over the sensor, the magnetic shadow accompanied by a concentration of flux lines. There is decreased in flux to the sides of the vehicle and increased flux above and below the vehicle [1]. The magnetometer installed within the earth sensed a decrease and increase of flux below the vehicle and this explains the inverted and noninverted pulses detected. The magnetic field variation or wavelet indicates the localization of a vehicle passes over the magnetometer as modeled in a previous report [18] without considering the direction of the object movement direction interrupting different vector fields.

Figure 8 shows the result of the Z-field or magnetic wavelets determined when a car passed over the magnetometer sensor node in counter/in-line X-axis and counter/in-line Y-axis of the magnetometer at 30 $\mathrm{kmph}$. Since the sensor node will be planted at the yellow box at the traffic light, $30 \mathrm{kmph}$ is the typical speed of the vehicle passing through the traffic light. The result shows that the sensitivity of the magnetometer sensor is dependent on the sensing direction of the sensor. Stronger Z-field from -100 to -230 $\mu \mathrm{T}$ were generated when a vehicle passed over the sensor in the counter $\mathrm{X}$ and $\mathrm{Y}$ axes. The current system detects Z-field in a 3-axis measurement in comparison to the 2-axis measurement which was based on the $\mathrm{Y}$ field detection [1,3]. The magnetic fields spread out in an omnidirectional manner [14]. The typical characteristic of the magnetic wavelets is either having single or two positive peaks and a negative dip when a ferrous magnetic material passed over the magnetometer. The negative dip is usually characterized by higher magnetic of density in comparison to the positive peak. The default Z-field at approximately $100 \mu \mathrm{T}$ is actually the quiescent magnetic field of earth in a 3-axis vector [15]. 


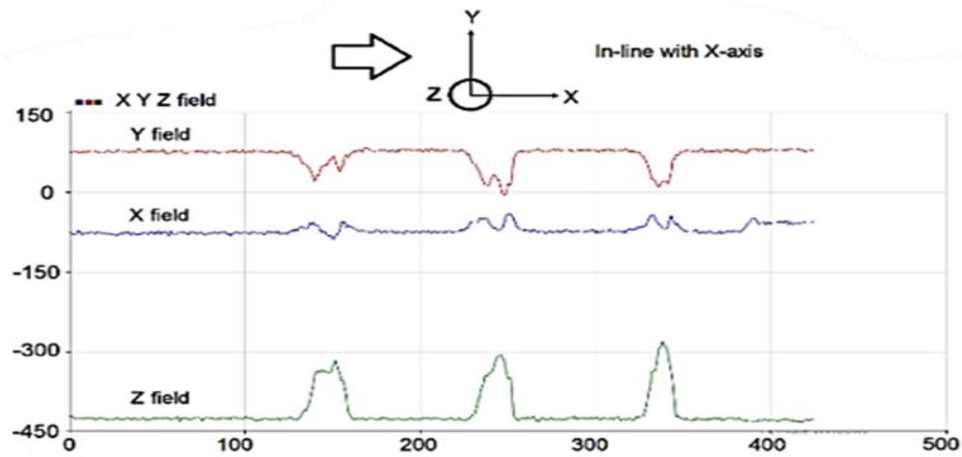

(a)

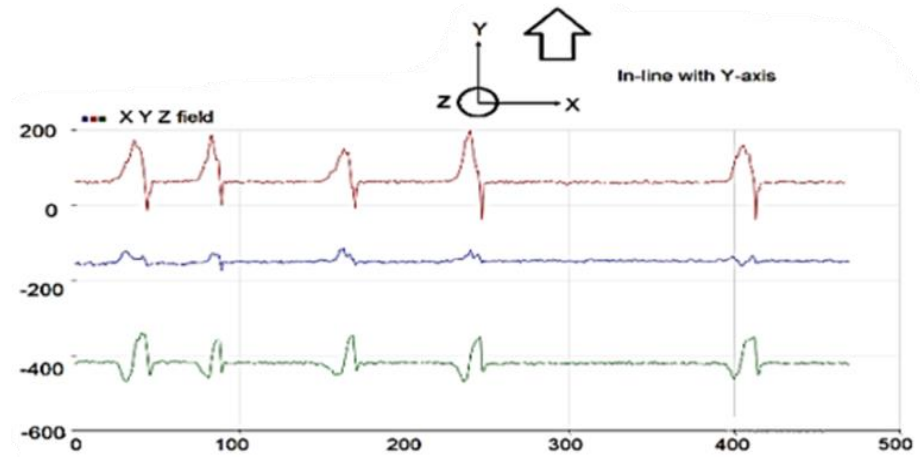

(b)

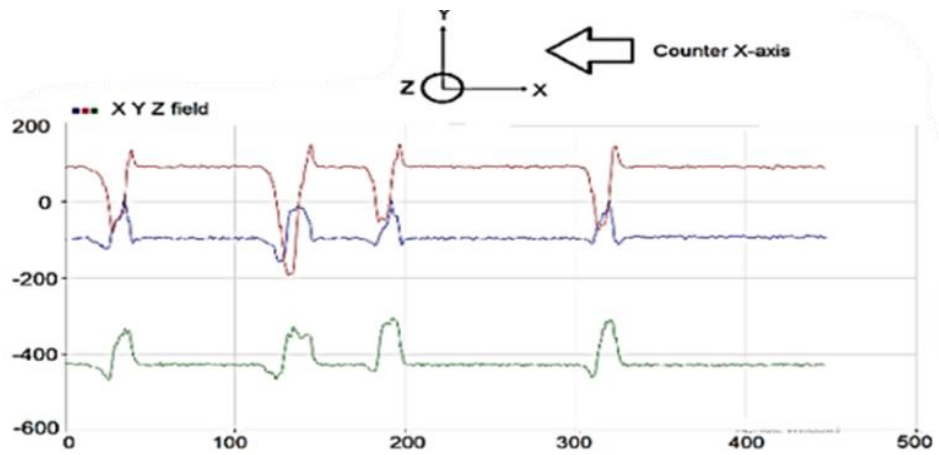

(c)

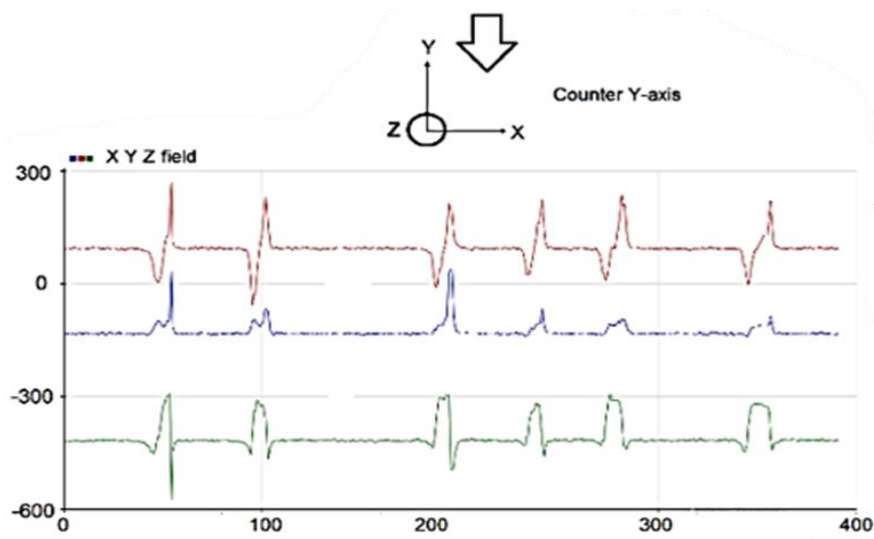

(d)

Figure 7. Responses of magnetometer with a ferrous object passing over. The thick arrows indicate the directions of the iron-rod movement in (a) in-line with $\mathrm{X}$-axis, (b) in-line with $\mathrm{Y}$-axis, (c) counter $\mathrm{X}$-axis, (d) counter Y-axis 
The Z-field determined for the iron bar and vehicle moving in counter $\mathrm{X}$ and $\mathrm{Y}$ axes were found different because of the condition of testing. In the test using the iron bar, the quiescent field measured by the magnetometer was approximately from -300 to $-400 \mu \mathrm{T}$ because the iron bar was a few millimeters away from the sensor Figure 7. Moreover, the magnetometer was placed on a workbench which contrasted with the magnetometer planted underground. For vehicle sensing, the sensing distance between the sensor and the car is approximately $50 \mathrm{~cm}$ and yielded Z-field between -100 and $-230 \mu \mathrm{T}$ Figure 8 . Non-inverted and inverter pulses of Z-field were generated for the test with iron bar and vehicle, respectively. The low speed of moving the iron rod (small ferrous object) over the magnetometer sensor creates a non-inverted pulse for the Z-field Figure 7, while large ferrous vehicle moving at a higher speed creates a strong inverted pulse for Z-field as shown in Figure 8. This was associated with the geometry and shape of the ferrous object passes over the magnetometer. Hence, the test with the magnetometer sensor planted underground reveals more accurate representation of the $\mathrm{Z}$-field measured for vehicle.

The false detection when a car cuts a corner and crossing the wrong lane as reported in [10]. This was due to the crossed detection of the magnetic sensor when a car passing a lane unrelated to their route and got too close to the sensor. This study [10] did not include the report on investigation of the stimulation magnetic field associating with the direction of the car passage over the sensor and sensing range. Moreover, this system was not fixed with wireless modules but depended on the microprocessor to transmit the data through state line as reported in [10]. The result of our current study highlighted the importance of placing the sensor in a direction that could produce strong magnetic flux. In addition, Figure 7 indicates that the magnetic field produced is not a simple inverted or non-inverted pulse but dependent on the direction of the car passed over. The biphasic magnetic field generated Figure 7 may be set as a condition in the algorithm to avoid false detection of car coming in opposite direction or double counting of long trailer as reported in [10]. A previous study [19] indicated the requirement of using two magnetic sensors embedded at the shoulder of the road for vehicle detection which is less efficient due to the weak magnetic field perturbation $(17 \mu \mathrm{T})$ and more sensors used. Similar magnetic sensor was used in sensing the intrusion of self-driving vehicle but the magnetic field changes in response to car intrusion direction were not well characterized and investigated [20].

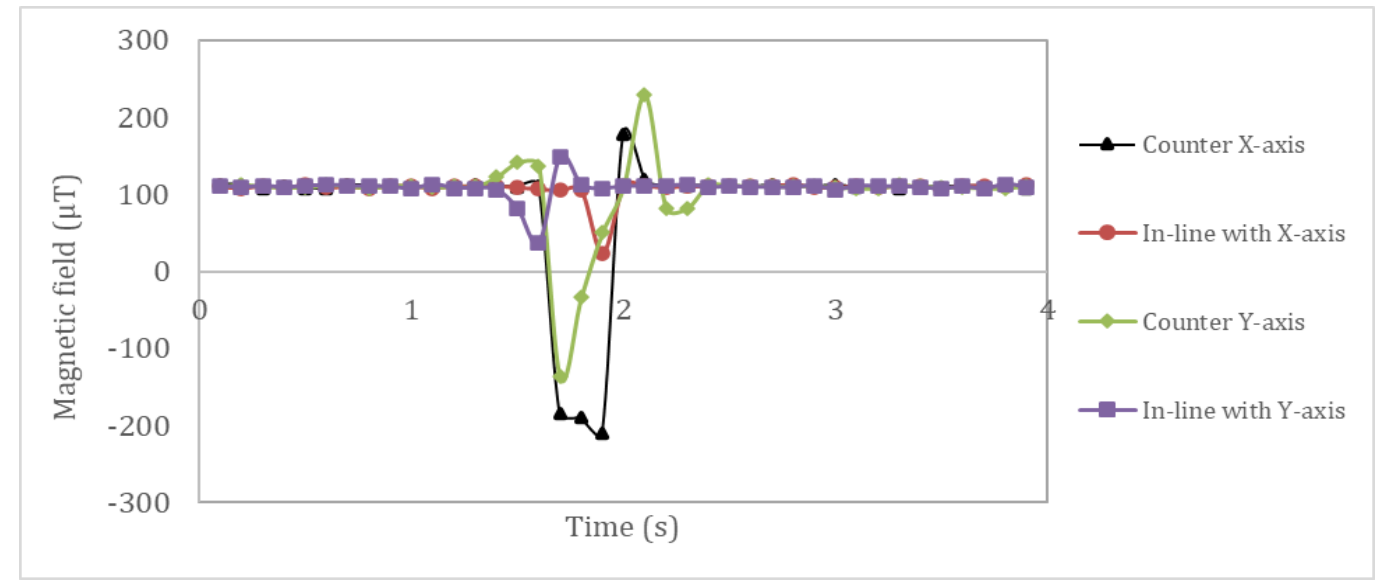

Figure 8. Magnetic field determined in Z-axis for a car passing over an electronic magnetometer in counter/in-line with $\mathrm{X}$-axis and counter/in-line with Y-axis of the measurement coordinate system of magnetometer at $30 \mathrm{kmph}$

The initial values for the four configurations of measurements were at approximately $100 \mu \mathrm{T}$. When the car passed over the sensor, the magnetic field dipped below $100 \mu \mathrm{T}$. After the car body passed the sensor, the magnetic field bounced back to positive values, returning to the initial value within a time frame of 800 ms. This is a typical wavelet modeled in [18]. However, among the four configuration of magnetic field measurement, the results showed that directions of the car pass over the magnetometer that are in-line with $\mathrm{X}$ and Y-axes yielded with low amplitude of magnetic flux. Contrarily, a huge inverted cycle of pulse dipped down to $-230 \mu \mathrm{T}$ was recorded for the magnetic field measurement in the counter $\mathrm{X}$ and $\mathrm{Y}$-axes. The observed results suggested that the placement of the magnetometer in certain direction would influence the density of the magnetic flux measured and should be considered carefully for practical applications on the 
road. The magnetometer may provide different level of initial value for the magnetic field when similar magneto-sensor node was placed in different locations. However, large magnetic field were formed when the vehicle was moving through in the counter $\mathrm{X}$ and $\mathrm{Y}$-axes. Low magnetic field detected could be used as a reference for classifying vehicle driving in the opposite direction [3, 21] and further applied for traffic surveillance. However, measuring the changes or drift of the flux during different time of the day, temperature [22] and different surfaces [23, 8] in detecting vehicle is beyond the scope of the current work.

For vehicle such as a car is a huge piece of solid ferrous metal consisting of complex magnetic flux generated by the engine within the chassis of the car. The results in Figure 9 indicates that the oscillations of the engine induced fluctuation of magnetic flux when a car is stationary on the magnetometer sensor node. The engine of a car is constructed from electromagnetic motors of multi-coils. When a vehicle is positioned over the magnetometer, the vibrating electromagnetic motors would repeatably increase and decrease flux of the magnetometer and hence, inducing fluctuating magnetic field in the inverted region of field density as observed. If the algorithm for measuring the number of vehicles is based on detecting the inverted pulses of the magnetic field, the multi inverted pulses generated might be mis-interpreted by the algorithm as false detection of vehicle. Base on this result, it is suggested to plant the magnetometer sensor node in the yellow box at the traffic light, in which the vehicle is prohibited from halting over the yellow box lines. Hence, the accuracy of counting the vehicle using the magnetometer can be ensured.

This study further investigated the effect of the sensitivity of the magnetometer sensor node in detecting car passes over the senor at different speed. At $5 \mathrm{kmph}$, the magnetic field was characterized by high amplitudes of density and broadly spread across the measurement time as indicated in Figure 10. Comparatively, smaller magnetic wavelets were generated for car speed tested for 5, 10, 20, 30, 40, 50 and $60 \mathrm{kmph}$. At highly congested area, 5-60 kmph is the range of car speed usually applied in passing the traffic light. The density of the magnetic field is related to the duration of interaction between the ferrous object perturbating the magnetic field formed over the magnetometer. Similar peaks and valleys of magnetic density could be observed despite of the car speed between $10-60 \mathrm{kmph}$. This range of car speed was selected for the test due to the consideration of normal car speed allowed at the traffic junction. Magnetic field measured was diminishing when the car speed is $>60 \mathrm{kmph}$ which is the detection limit of the magnetometer sensor. The data determined for different car speed using magnetometer could also be applicable for car speed detector and surveillance as reported in [24, 25].

This study further investigated the effect of the sensitivity of the magnetometer sensor node in detecting car passes over the senor at different speed. At $5 \mathrm{kmph}$, the magnetic field was characterized by high amplitudes of density and broadly spread across the measurement time as indicated in Figure 10. Comparatively, smaller magnetic wavelets were generated for car speed tested for 5, 10, 20, 30, 40, 50 and $60 \mathrm{kmph}$. At highly congested area, $5-60 \mathrm{kmph}$ is the range of car speed usually applied in passing the traffic light. The density of the magnetic field is related to the duration of interaction between the ferrous object perturbating the magnetic field formed over the magnetometer. Similar peaks and valleys of magnetic density could be observed despite of the car speed between $10-60 \mathrm{kmph}$. This range of car speed was selected for the test due to the consideration of normal car speed allowed at the traffic junction. Magnetic field measured was diminishing when the car speed is $>60 \mathrm{kmph}$ which is the detection limit of the magnetometer sensor. The data determined for different car speed using magnetometer could also be applicable for car speed detector and surveillance as reported in [24, 25].

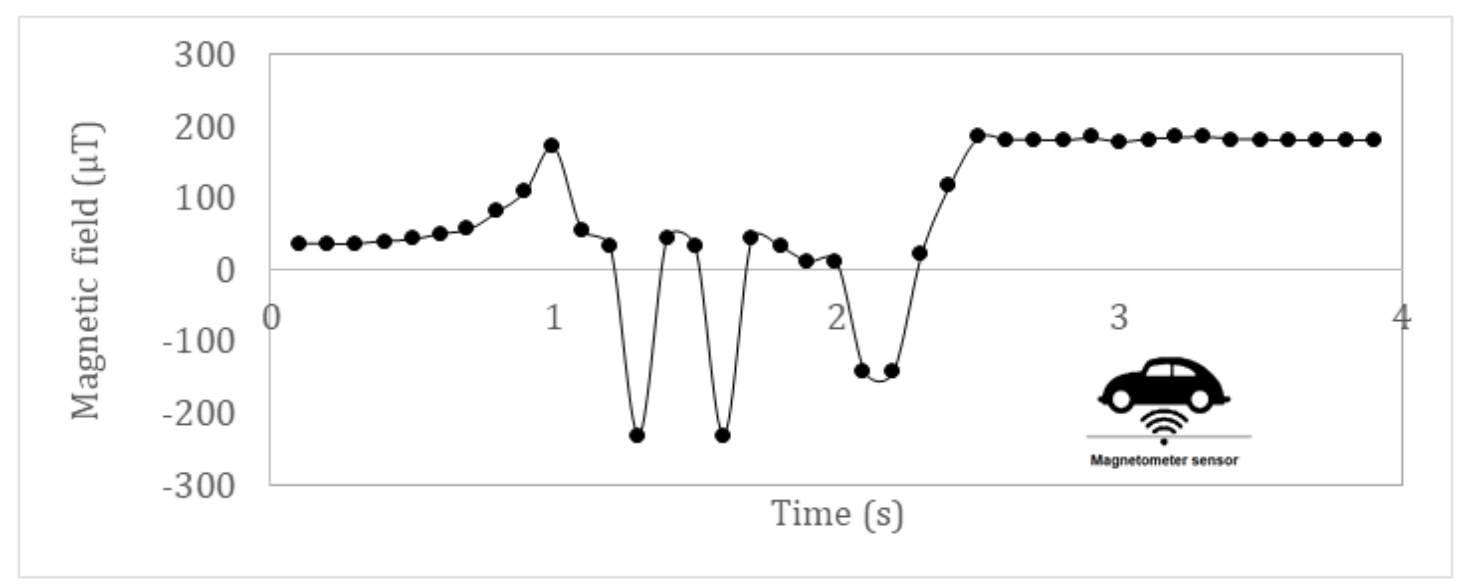

Figure 9. Magnetic field in Z-axis for a car halting over the electronic magnetometer sensor 


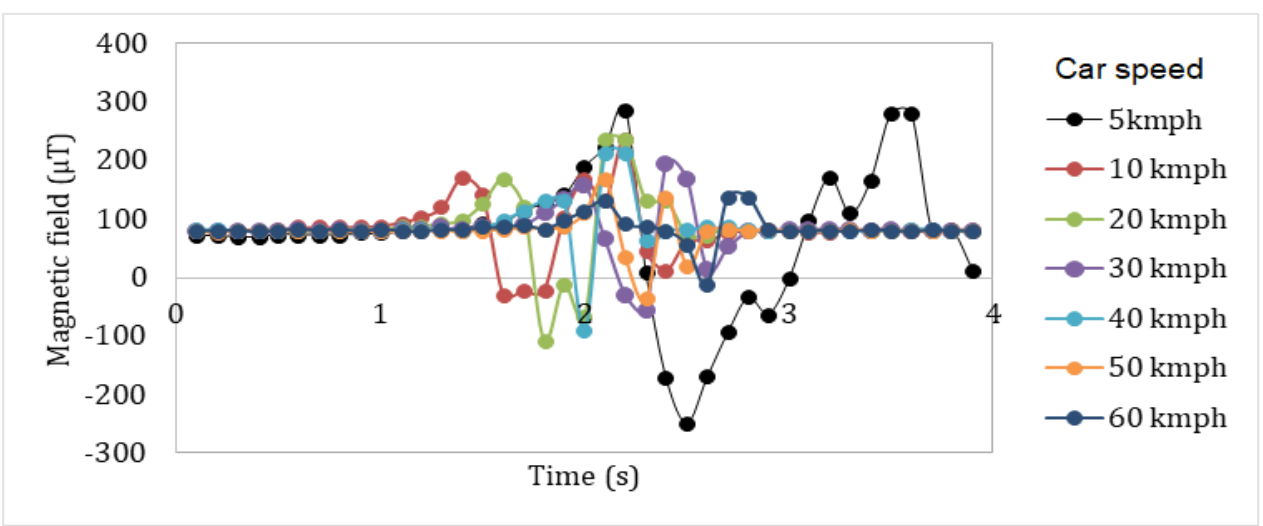

Figure 10. Magnetic field determined for different car speed ranging from 5 to $60 \mathrm{kmph}$

The advantage of the magnetometer sensor over the induction loop is the simplicity in deployment without the complex calculation of inductance and installation of the induction loop. Minimum invasiveness to the road is required for embedding the magnetometer compared with the induction loop installation. The wireless magnetometer sensor can continuously transmit data every $200 \mathrm{~ms}$ after sensing.

\section{CONCLUSION}

A wireless magnetometer sensor node and gateway for recording the measured magnetic field strength and direction have been developed for vehicle detection. After sensing, the vehicle volume detected will be transmitted to gateway every $200 \mathrm{~ms}$. The system has been developed to detect dynamic ferrous object efficiently and reliably. The flux pattern determined for $\mathrm{X}, \mathrm{Y}$ and $\mathrm{Z}$ fields were characterized by inverted, non-inverted or biphasic pulses, depending on the direction of the ferrous object in perturbating the coordinate system of the magnetometer sensor. The flux patterns measured on workbench using an iron rod was seen different from the measurement of flux patterns produced by a vehicle, which is due to the size and speed of the ferrous object passing over the magnetometer. The wireless sensor node system transmitted magnetic field signals detected at radio frequency of $2.4 \mathrm{GHz}$ to a gateway located at approximately 10 meters away. The magnetometer measurement is directional dependent in which, the car passes over the magnetometer sensor in countering the $\mathrm{X}$ - and $\mathrm{Y}$ - axes of the sensor generated stronger magnetic field density from -100 to $-230 \mu \mathrm{T}$. This information is important in designing the vehicle detection algorithm whenever a vehicle is passes over the sensor. However, a car is not suggested to be halting on the sensor node due to the generation of multi inverted pulses of magnetic field which could be misleading to false detection of vehicle. It is suggested to place the sensor node in the yellow box region where vehicle is forbidden from stopping over. The car speed is an influencing factor to the sensitivity of the magnetometer. A ferrous object passes at high speed $>60 \mathrm{kmph}$ would not be detectable by the magnetometer due to the response time of the sensor. This work has successfully identified modulations of magnetic field measured by a wireless magnetometer sensor node with respect to the different axis of measurement.

\section{ACKNOWLEDGEMENTS}

The authors are grateful for the financial support from Vot. No. K233 awarded by Malaysia Ministry of Higher Education (KPT) and Malaysia Technical University Network (MTUN), Universiti Tun Hussein Onn Malaysia and SENA Traffic System Sdn. Bhd. The assistance from our undergraduate student, Priscilla Poh Swee Pei during the field test is appreciated.

\section{REFERENCES}

[1] Lawrence A., K., M. Milton. K., and G. David. R, "Traffic detector handbook," Federal Highway Administration, Turner-Fairbank Highway Research Center, Washington, DC, USA: Federal Highway Admin, 2006.

[2] Mit, K. S., N. S. Parshva, M. T. Khyati, and R. S. Smita, "Vehicle monitoring and routing system," International Journal of Computer Applications, vol. 138, no. 12, pp. 7-11, 2016. 
[3] N. Wahlström, R. Hostettler, F. Gustafsson and W. Birk, "Classification of Driving Direction in Traffic Surveillance Using Magnetometers," in IEEE Transactions on Intelligent Transportation Systems, vol. 15, no. 4, pp. 1405-1418, Aug. 2014.

[4] Panfeng, Z., M. Lingkun, and L. Huizhen, "Design of Wireless Mine Gas Monitoring and Control System Based on nRF2401," 2012 International Conference on Computer Science and Service System, Nanjing, pp. 1051-1054, 2012.

[5] A. Sharma, R. Chaki and U. Bhattacharya, "Applications of wireless sensor network in Intelligent Traffic System: A review," 2011 3rd International Conference on Electronics Computer Technology, Kanyakumari, pp. 53-57, 2011.

[6] Transportation, U. S. D. o., "Traffic Safety Facts 2010: A Compilation of Motor Vehicle Crash Data From the Fatality Analysis Reporting System and the General Estimates System," 2010 Motor Vehicle Crash Data from FARS and GES, 2010.

[7] Somashekhar, G. C., S. Shirabadagi, and R. S. Hegadi. "High density traffic management using Image background subtraction algorithm," International Journal of Computer Applications, vol. 4, pp. 10-15, 2014.

[8] Orfeo, D., D. Burns, R. Farrell, M. Qin, H. Mitchell, C. Ou, T. Xia, and R. H. Dryver, "Mechano-magnetic telemetry for underground water infrastructure monitoring," Frontiers in Built Environment Structural Sensing, vol. 14, no. 29 , pp. 1-14, 2018.

[9] X. Zhang and H. Huang, "Vehicle Classification Based on Feature Selection With Anisotropic Magnetoresistive Sensor," in IEEE Sensors Journal, vol. 19, no. 21, pp. 9976-9982, 1 Nov.1, 2019.

[10] Bugdol, M., Z. Segiet, M. Krecichwost, and P. Kasperek, "Vehicle detection system using magnetic sensors," Transport Problems, vol. 9, no. 1, pp. 49-60, 2014.

[11] M. J. Caruso, "Applications of magnetic sensors for low cost compass systems," IEEE 2000. Position Location and Navigation Symposium (Cat. No.00CH37062), San Diego, CA, USA, pp. 177-184, 2000.

[12] C. Song and K. Wen, "Integration Design of Wide-Dynamic-Range MEMS Magnetometer and Oscillator," 2018 IEEE International Conference on Semiconductor Electronics (ICSE), Kuala Lumpur, pp. 17-20, 2018.

[13] A. Grosz, E. Paperno, S. Amrusi and B. Zadov, "A Three-Axial Search Coil Magnetometer Optimized for Small Size, Low Power, and Low Frequencies," in IEEE Sensors Journal, vol. 11, no. 4, pp. 1088-1094, April 2011.

[14] Matsuoka, A., M. Shinohara, Y.-M. Tanaka, A. Fujimoto, and K. Iguchi, "Development of fluxgate magnetometers and applications to the space science missions," An Introduction to Space Instrumentation, pp. 217-224, 2013.

[15] McCaughey, M, “An Arduino-Based Magnetometer,” The Physics Teacher, vol. 55, no. 5, pp. 274-275, 2017.

[16] Q. Wang, J. Zheng, H. Xu, B. Xu and R. Chen, "Roadside Magnetic Sensor System for Vehicle Detection in Urban Environments," in IEEE Transactions on Intelligent Transportation Systems, vol. 19, no. 5, pp. 1365-1374, May 2018.

[17] P. Sarcevic and S. Pletl, "False Detection Filtering Method for Magnetic Sensor-Based Vehicle Detection Systems," 2018 IEEE 16th International Symposium on Intelligent Systems and Informatics (SISY), Subotica, pp. 000277-000282, 201

[18] N R. Hostettler and P. M. Djurić, "Vehicle Tracking Based on Fusion of Magnetometer and Accelerometer Sensor Measurements With Particle Filtering," in IEEE Transactions on Vehicular Technology, vol. 64, no. 11, pp. 49174928, Nov. 2015.

[19] N. Wahlström and F. Gustafsson, "Magnetometer Modeling and Validation for Tracking Metallic Targets," in IEEE Transactions on Signal Processing, vol. 62, no. 3, pp. 545-556, Feb 2014.

[20] K. M. Ali Alheeti, A. Gruebler and K. D. McDonald-Maier, "An intrusion detection system against malicious attacks on the communication network of driverless cars," 12th Annual IEEE Consumer Communications and Networking Conference (CCNC), Las Vegas, NV, pp. 916-921, 2015.

[21] N. Wahlström, R. Hostettler, F. Gustafsson and W. Birk, "Rapid classification of vehicle heading direction with two-axis magnetometer," IEEE International Conference on Acoustics, Speech and Signal Processing (ICASSP), Kyoto, pp. 3385-3388, 2012.

[22] Malátek, M., P. Ripkaa, and L. Krausb, "Temperature offset drift of gmi sensors," Sensors and Actuators A: Physical, vol. 147, no. 2, pp. 415-418, 2008.

[23] P. Nasta et al., "Integrating ground-based and remote sensing-based monitoring of near-surface soil moisture in a Mediterranean environment," IEEE International Workshop on Metrology for Agriculture and Forestry (MetroAgriFor), Portici, Italy, pp. 274-279, 2019.

[24] Bernas, M., B. Płaczek, W. Korski, P. Loska, J. Smyła, and P. Szymała. "A survey and comparison of low-cost sensing technologies for road traffic monitoring," Sensors (Basel), vol. 18, no. 10, pp. 1-27, 2018.

[25] Nuryantini, A. Y., A. Sawitri, and B. W. Nuryadin, "Constant speed motion analysis using a smartphone magnetometer," Physics Education, vol. 53, no. 6, 2018. 